\title{
Event-related potentials (ERPs) and hemodynamic (functional near-infrared spectroscopy, fNIRS) as measures of schizophrenia deficits in emotional behavior
}

\author{
Michela Balconi ${ }^{1,2 *}$, Simone Tirelli ${ }^{2}$ and Alessandra Frezza ${ }^{2,3}$ \\ ${ }^{1}$ Research Unit in Affective and Social Neuroscience, Catholic University of the Sacred Heart, Milan, Italy, ${ }^{2}$ Department of \\ Psychology, Catholic University of the Sacred Heart, Milan, Italy, ${ }^{3}$ Sapienza University of Rome, Rome, Italy
}

OPEN ACCESS

Edited by:

Salvatore Campanella, Université Libre de Bruxelles, Belgium

Reviewed by:

Thibaut Dondaine,

Ghent University, Belgium Giulia Bucchioni,

Université de Picardie Jules Verne,

France

*Correspondence. Michela Balconi michela.balconi@unicatt.it

Specialty section:

This article was submitted to

Psychopathology,

a section of the journal

Frontiers in Psychology

Received: 25 August 2015 Accepted: 19 October 2015 Published: 03 November 2015

Citation:

Balconi M, Tirelli S and Frezza A (2015) Event-related potentials (ERPS)

and hemodynamic (functional near-infrared spectroscopy, fNIRS) as measures of schizophrenia deficits in emotional behavior.

Front. Psychol. 6:1686. doi: 10.3389/fpsyg.2015.01686
Recent research evidences supported the significant role of multimethodological neuroscientific approach for the diagnosis and the rehabilitative intervention in schizophrenia. Indeed both electrophysiological and neuroimaging measures in integration each other appear able to furnish a deep overview of the cognitive and affective behavior in schizophrenia patients (SPS). The aim of the present review is focused on the emotional dysfunctional response taking into account the multimeasures for emotional behavior, i.e., the event-related potentials (ERPs) and the hemodynamic profile functional near-infrared spectroscopy (fNIRS). These measures may be considered as predictive measures of the SPs' deficits in emotional behavior. The integration between ERP and fNIRS may support both the prefrontal cortical localization anomaly and the attentional bias toward some specific emotional conditions (mainly negative).

Keywords: schizophrenia, NIRS, emotion, ERPs, integration

\section{EMOTIONAL IMPAIRMENT IN SCHIZOPHRENIA}

Affective deficits are considered to be a core feature of schizophrenia (Kring and Elis, 2013). Emotional deficits in schizophrenia affect diverse processes, including emotional experience (Myin-Germeys et al., 2000; Blanchard and Cohen, 2006; Horan et al., 2008; Cohen and Minor, 2010; Taylor et al., 2012), emotion expression (Blanchard and Cohen, 2006), and perception and recognition of emotional cues (Couture et al., 2006; Hoekert et al., 2007; Kohler et al., 2010; Horan et al., 2011). In contrast, other emotional skills, such as the ability to appraise the emotional valence of stimuli, appear relatively preserved in schizophrenia (Kring and Moran, 2008). However in some cases inconsistent data were collected (Strauss et al., 2011). Indeed, from one hand, recent evidences on schizophrenia impairment in emotional behavior were based upon the observation that patients displayed "blunted" or "flat" facial and vocal affect, which was supposed to reveal a correspondingly diminished emotional experience. From the other hand, available data suggests that their subjective experience of emotion is not consistently modified (Aghevli et al., 2003; Herbener et al., 2007, 2008). Further, previous studies have indicated that patient self-report of affective experience has high internal consistency (Blanchard et al., 1998; Horan et al., 2006a,b). Moreover, a number of studies have found that patients do not differ from healthy subjects in their ratings of a variety of 
affective stimuli with regard to valence and arousal ratings (Kring et al., 2003; Kring and Moran, 2008; Cohen and Minor, 2010). In general, results did not support a primary hedonic deficit in schizophrenia (for example, rating positive stimuli as less pleasant). Rather, data suggested that atypical emotional response is mostly associated with experiencing negative stimuli as being highly unpleasant and arousing. These potential contrasting effects can be also interpreted in terms of two broad social cognitive processes: lower-level versus higherlevel (Frith and Frith, 2006; Ochsner et al., 2009; Green and Lee, 2012). Lower-level are social cognitive processes which involve recognizing significance in social and emotional stimuli (such as recognition of facial expression of emotions and vocal emotional features). These processes are considered as partially automatic neural responses to emotional cues and usually rely on specific neural circuits, including amygdala and insula. Higher-level processes involve an ability to make inferences about or contextualize representations that are driven by lowlevel recognition processes. These two levels may be partially preserved but they may have some difficulties to be connected, generating discordances between emotion experience, expression and recognition (Regan et al., 2015).

In the present review we mainly focused on emotional cue perception and recognition. Indeed emotion perception is one of the most relevant component of social cognition in schizophrenia and it usually involves the identification of emotions displayed in emotional patterns or in facial stimuli.

Electrophysiology (EEG) and functional Magnetic Resonance (fMRI) approaches are able to examine neural correlates for explicit as well as implicit tasks (i.e., the brain response to emotional faces during a gender identification task). It is reasonable to infer that brain imaging is appropriate for identifying various impairments in schizophrenia which are localized brain areas (Champagne et al., 2014). Nevertheless, it is limited by its low temporal resolution that does not support the real-time dynamic of the emotional filed (Logothetis, 2008). In contrast, event related potentials (ERPs), provide rate which allows milliseconds accuracy in exploring cerebral activation and a vast amount of research has already underlined its validity with emotional stimuli (Olofsson et al., 2008). Indeed, although somewhat contrasting, in general the findings from rating scales and EEG show lack of differences between schizophrenia patients (SPs) and healthy subjects: patients appear to recognize emotional stimuli to the same extent as healthy persons, even though in some cases they rate higher on anhedonia scales.

\section{ERPS AS MEASURE OF SCHIZOPHRENIA EMOTIONAL DEFICITS}

Event-related potential measure offers the advantage to monitor the dynamical modulations of the emotional processes, taking into account the heterogeneous levels implicated in emotional behavior. Indeed in the next paragraphs we considered ERP variation in response to emotional stimuli and facial expression patterns in SP.

\section{Early/Late ERPs Modulation in Emotional Tasks}

Many reviews underlined the utility to apply ERP measurement in emotional study and specifically to schizophrenia (Friedman and Squires-Wheeler, 1994). Indeed it was found to be suitable to measure changes in brain activity at early and late latencies, furnishing a complete overview of the emotional processing across-time. In fact, early and late mechanisms sequentially describe the dynamic variations of subjective response to emotional contexts. This dynamic modulations are not easily accessible by classical neuroimaging measures. Specifically, some ERP deflections were explored in emotional domain, such as P100, N100, P200, P300, and late positive component (LPC; Neuhaus et al., 2010). About the early latency and middle-latency deflections (P100, N100, P200, P300), the ERP data suggest that SP emotional experience are accompanied by normal early stimulus processing and initial resource allocation processes in response to emotional stimuli (Horan et al., 2010). A consistent set of data did not evidence of hypo- or hyper-responsivity to pleasant or unpleasant stimuli in SP among these three ERP components. The topography of P100, P200, and P300 responses was also similar across SP and healthy controls, who show maximal responses in central and parietal regions of the right than left hemisphere. It was previously found that the early latency P100 is sensitive to perceptual stimulus features and indexes early sensory processing, whereas the middle-latency P200 and P300 demonstrated to be highly sensitivite to valence. The P200 response reflects early stimulus discrimination and response selection processes: it was increased in response to emotional pictures reflecting a relatively automatic attentional allocation by emotionally more arousing stimuli. The enhanced P300 amplitude to emotional stimuli is thought to reflect greater allocation of attention to emotionally relevant stimuli.

Therefore these previous results seemed to underline the quite similar profile of SP and control subjects in these early and middle- time response to emotions. However it was also found that emotionally evocative stimuli are differentially processed in SP within the first time interval of $200 \mathrm{~ms}$, and, for this reason, that the early stages of emotional process may be affected (Pinheiro et al., 2013; Champagne et al., 2014). Furthermore, some recent study underlined differences in SP in later components, observing significant difference in the LPC in response to unpleasant pictures (Strauss et al., 2013). This could be due to a possible inability to downregulate emotional response by SP in a later phase of emotion processing. Specifically, about the more later potentials, a higher agreement was obtained across the studies, since the patients differed from controls in terms of valence-related amplitude and topography during the late-latency LPC interval. As in prior studies (Olofsson et al., 2008), controls showed clearly enhanced LPC amplitudes for pleasant and unpleasant as compared to neutral stimuli since LPC enhancement is believed to index sustained attentional processing of motivationally relevant stimuli (Bradley and Lang, 1994). However, the patients showed significantly less LPC differentiation between emotional (mainly pleasant) versus neutral stimuli. Hence, the pattern of results for LPC may suggest a disturbance in sustained attentional processing of pleasant 
stimuli in schizophrenia. In some cases LPC modulation also indicated that there was a difference in laterality between SP and control healthy subjects. Controls generally showed greater left than right hemisphere responses for pleasant stimuli whereas patients did not demonstrate any specific laterality (Dolcos and Cabeza, 2002; Cunningham and Zelazo, 2007). The left more than right asymmetry of LPC in controls is consistent with neuropsychological models that propose a significant left hemisphere specialization for pleasant emotion processing in frontal regions (Heller and Nitschke, 1998; Davidson and Irwin, 1999; Balconi et al., 2009; Balconi and Mazza, 2011; Balconi et al., 2012). Therefore these studies pointed out the significance that the lateralization effect may have to explain the emotional behavior disturbance.

It was also suggested the SP' pattern of intact ERPs during initial processing stages but diminished differentiation between emotional versus neutral stimuli during the LPC points toward a disturbance in "affective chronometry." This concept refers to parameters that vary over the time course of emotional behavior (Davidson, 1998). In fact, the earlier components may be represented as more stimulus-driven processes, and the later components as involving more cognitive evaluation and controlled resources. Consistent with this view, recent studies have shown that these later ERP components are regulated by top-down attentional mechanisms (Hajcak and Nieuwenhuis, 2006; Moser et al., 2006; Hajcak and Foti, 2008).

\section{ERP Evidences for Deficit in Emotion "Regulation" and Attention Disengagement in SP}

There previous results on ERPs may support the hypothesis that SP would show an impairment in emotion regulation. Emotion regulation refers to the processes by which we modify negative and positive emotions in terms of intensity, duration, and how they are manifested. This conceptualization is based on the idea that emotions unfold as a multicomponential process, which can be regulated by using subjective strategies at different stages of emotion generation. The topic of emotion dysregulation may be explored focusing on the "negative impairment effect" found in many previous studies, which underline the anomalous response to negative patterns by SP. In fact one possible explanation for these increases in state and trait negative emotionality is that SP display impairments in "emotion regulation" (Cohen and Minor, 2010; Cohen et al., 2011; Horan et al., 2011; Strauss et al., 2011, 2013; Strauss and Gold, 2012), since cognitive change is associated with increased prefrontal cortex activity and decreased amygdala activity (Ochsner et al., 2002, 2004, 2012; Ochsner and Gross, 2005; Mocaiber et al., 2011), it is possible that the concomitant ERP findings reflect that SP either have ineffective cortical control over the amygdala and limbic regions or a failure to adequately engage prefrontal and limbic regions when applying cognitive change strategies to perform emotional task.

From another point of view, the ERP measures are usable to verify if SP had greater difficulty disengaging attention from unpleasant stimuli (Strauss et al., 2011). Indeed this impairment may play a critical role in the generation of both negative symptoms and trait negative affect. Problems with disengaging attention from unpleasant stimuli have been proposed to be a major contributor to elevated negative emotional experience in individuals with other psychiatric disorders (e.g., anxiety) (Fox et al., 2001), and SP as well (Kring and Moran, 2008; Cohen and Minor, 2010). An enhanced in negative emotion response may reflect a sustained emotional regulation problem, where patients have difficulty regulating negative mood and negative behavior (Horan et al., 2006a,b; Cohen and Minor, 2010; Cohen et al., 2011).

The present findings are consistent with prior studies demonstrating a sort of disconnection among emotional response subcomponents in SP. For example, it is well documented that SP are less expressive than healthy controls, yet do not differ as much with respect to reported emotional experience or autonomic physiology (Kring and Moran, 2008). Failure to sufficiently process motivationally relevant stimuli could have maladaptive functional consequences for SP (Dolcos and Cabeza, 2002). Diminished attentional processing reflected in the LPC could help explain why SP fail to show the memory enhancements for emotional patterns.

\section{Impairment in Facial Expression Comprehension}

A deficit in the recognition of facial emotion is well established in SP (Walker et al., 1984; Archer et al., 1992; Schneider et al., 1995; Salem et al., 1996; Addington and Addington, 1998; Kee et al., 1998; Kohler et al., 2003; Johnston et al., 2005). This deficit appears to be at least partly related to a more general problem in cognitive functions including the categorization, discrimination, and identification of facial stimuli, and also to deficits in working memory and attentional processes (Addington and Addington, 1998; Kee et al., 1998; Kohler et al., 2000). It was revealed that anomalous late-latency activity was related to specific attentional process to facial expression, such as an attenuation of P300 response to different emotional valences (An et al., 2003). Moreover recent evidences were reported for impaired information processing in schizophrenia that occurs at the encoding level of facial stimuli. The findings in the emotion perception analysis probably reveal that SP do not modulate the amygdala to emotional versus non-emotional faces to the same extent as controls. It was also shown that SP exhibited amplitude deficits for both the late components N170, related to processing of structural components of face, and N250, related to the emotional content processing, but a latency deficit only for the N250 (Wynn et al., 2013).

\section{INCOMING DEVELOPMENTS: WHY TO USE FUNCTIONAL NEAR-INFRARED SPECTROSCOPY (fNIRS) IN SCHIZOPHRENIA STUDY ON EMOTIONS}

Although the good temporal resolution of ERP enables a precise evaluation of the time-course of neural response in response 
to the emotion perception, its low spatial resolution makes less easy to draw accurate conclusions regarding the main neural areas involved. Therefore, the neural substrates responsible for these emotion abnormalities cannot be definitively determined using ERP alone. For this reason, recently fNIRS measure was applied to study emotions in schizophrenia (Koseki et al., 2013). fNIRS has been developed to be noninvasive, easy-to-use, portable, restraint-free, and replicable (Kono et al., 2007). Indeed fNIRS is considered to impose considerably milder physical and psychological burdens than those of classical neuroimaging techniques. It is less invasive than other techniques (for example it does not require injection of radioactive agent), and no side effects are described so far and it is thus suitable for children and patients.

Indeed fNIRS is a functional brain imaging methodology from among other available methodologies such as fMRI and positron emission tomography (PET). While both of them also have excellent spatial resolution, fMRI and PET require uneasy apparatus. In contrast, fNIRS is as portable devices which allows to use it in various and critical conditions, as studies with SP (Matsuo et al., 2003). In addition, the high temporal resolution of ANIRS is useful in characterizing the time course of prefrontal activity of psychiatric pathologies (Suto et al., 2004; Kameyama et al., 2006).

In addition, patients are examined in a normal sitting position in a quiet place without any disturbing noise and examination cost is much lower than other neuroimaging modalities. However, fNIRS also has some limitations: poor spatial resolution, inability to measure deeper cerebral structures, and the possibility of involvement of extra-cerebral structures such as the scalp, fact which makes the validity of fNIRS results to be better evaluated in the future.

Previously fNIRS has been applied to assess brain functions of patients with psychiatric disorders such as schizophrenia, bipolar

\section{REFERENCES}

Addington, J., and Addington, D. (1998). Facial affect recognition and information processing in schizophrenia and bipolar disorder. Schizophr. Res. 32, 171-181. doi: 10.1016/S0920-9964(98)00042-5

Aghevli, M. A., Blanchard, J. J., and Horan, W. P. (2003). The expression and experience of emotion in schizophrenia: a study of social interactions. Psychiatry Res. 119, 261-270. doi: 10.1016/S0165-1781(03)00133-1

An, S., Lee, S., and Lee, C. (2003). Reduced P3 amplitudes by negative facial emotional photographs in schizophrenia. Schizophr. Res. 64, 125-135. doi: 10.1016/S0920-9964(02)00347-X

Archer, J., Hay, D., and Young, A. (1992). Face processing in psychiatric conditions. Br. J. Clin. Psychol. 31, 45-61. doi: 10.1111/j.2044-8260.1992.tb00967.x

Balconi, M., Falbo, L., and Brambilla, E. (2009). BIS/BAS responses to emotional cues: self-report, autonomic measure and alpha band modulation. Pers. Individ. Dif. 47, 858-863. doi: 10.1016/j.paid.2009.07.004

Balconi, M., Falbo, L., and Conte, A. V. (2012). BIS and BAS correlates with psychophysiological and cortical response systems during aversive and appetitive emotional stimuli processing. Motiv. Emot. 36, 218-231. doi: 10.1007/s11031-011-9244-7

Balconi, M., and Mazza, G. (2011). Lateralisation effect in comprehension of emotional facial expression: a comparison between EEG alpha band power disorder, depression, dementia, post-traumatic stress disorder, and pervasive developmental disorder (Fallgatter et al., 1997; Hock et al., 1997; Matsuo et al., 2004; Shinba et al., 2004; Suto et al., 2004; Kubota et al., 2005; Kameyama et al., 2006; Kuwabara et al., 2006). fNIRS was clinically applied for the assessment of psychiatric patients, reporting that the frontal lobe activation measured by fNIRS in SP during a verbal fluency task was lower than that in healthy controls (Okada et al., 1994).

Recent developments of studies on schizophrenia which used fNIRS measure has found emotional recognition impairment in SP and the multichannel fNIRS was shown to be a valid measure of this impaired functions (Shoji et al., 2013). In addition, schizophrenia has been shown to have neural network abnormalities in the social brain, which subserves social and interpersonal relationships (Mimura, 2014).

Therefore the integration between ERP and fNIRS measures may describe the main features of the emotional process, that is the dynamical evolution of such phenomenon (by both fNIRS and ERP) and the contribution by specific cortical areas in processing emotions (by fNIRS). No other integrated measures may respond so well to the nature of emotional behavior. However, due the limited number of studies which have used fNIRS actually applied to schizophrenia domain, the potentiality of this technique has to be explored in the next future.

\section{CONCLUSION}

As pointed out by previous research, both ERPs and fNIRS measures may well elucidate the cortical correlates and functional features of emotional processing. Allowing a good temporal and spatial resolution, they may furnish a complete overview of SP' deficits in emotional recognition. Moreover, their portability and easiness of application make them the favorite devices to describe the multicomponential domain of emotions.

and behavioural inhibition (BIS) and activation (BAS) systems. Laterality 15, 361-384. doi: 10.1080/13576500902886056

Blanchard, J. J., and Cohen, A. S. (2006). The structure of negative symptoms within schizophrenia: implications for assessment. Schizophr. Bull. 32, 238-245. doi: 10.1093/schbul/sbj013

Blanchard, J. J., Mueser, K. T., and Bellack, A. S. (1998). Anhedonia, positive and negative affect, and social functioning in schizophrenia. Schizophr. Bull. 24, 413-424. doi: 10.1093/oxfordjournals.schbul.a033336

Bradley, M. M., and Lang, P. J. (1994). Measuring emotion: the self-assessment manikin and the semantic differential. J. Behav. Ther. Exp. Psychiatry 25, 49-59. doi: 10.1016/0005-7916(94)90063-9

Champagne, J., Mendrek, A., Germain, M., Hot, P., and Lavoie, M. E. (2014). Eventrelated brain potentials to emotional images and gonadal steroid hormone levels in patients with schizophrenia and paired controls. Front. Psychol. 5:543. doi: 10.3389/fpsyg.2014.00543

Cohen, A. S., and Minor, K. S. (2010). Emotional experience in Patients with schizophrenia revisited: meta-analysis of laboratory studies. Schizophr. Bull. 36, 143-150. doi: 10.1093/schbul/sbn061

Cohen, A. S., Najolia, G. M., Brown, L. A., and Minor, K. S. (2011). The state-trait disjunction of anhedonia in schizophrenia: potential affective, cognitive and social-based mechanisms. Clin. Psychol. Rev. 31, 440-448. doi: 10.1016/j.cpr.2010.11.001 
Couture, S. M., Penn, D. L., and Roberts, D. L. (2006). The functional significance of social cognition in schizophrenia: a review. Schizophr. Bull. 32, S44-S63. doi: $10.1093 / \mathrm{schbul} / \mathrm{sbl} 029$

Cunningham, W. A., and Zelazo, P. D. (2007). Attitudes and evaluations: a social cognitive neuroscience perspective. Trends Cogn. Sci. 11, 97-104. doi: 10.1016/j.tics.2006.12.005

Davidson, R. J. (1998). Affective style and affective disorders: perspectives from affective neuroscience. Cogn. Emot. 12, 307-330. doi: $10.1080 / 026999398379628$

Davidson, R. J., and Irwin, W. (1999). The functional neuroanatomy of emotion and affective style. Trends Cogn. Sci. 3, 11-21. doi: 10.1016/S13646613(98)01265-0

Dolcos, F., and Cabeza, R. (2002). Event-related potentials of emotional memory: encoding pleasant, unpleasant, and neutral pictures. Cogn. Affect. Behav. Neurosci. 2, 252-263. doi: 10.3758/CABN.2.3.252

Fallgatter, A. J., Roesler, M., Sitzmann, L., Heidrich, A., Mueller, T. J., and Strik, W. K. (1997). Loss of functional hemispheric asymmetry in Alzheimer's dementia assessed with near-infrared spectroscopy. Brain Res. Cogn. Brain Res. 6, 67-72. doi: 10.1016/S0926-6410(97)00016-5

Fox, E., Russo, R., Bowles, R., and Dutton, K. (2001). Do threatening stimuli draw or hold visual attention in subclinical anxiety? J. Exp. Psychol. Gen. 130, 681-700. doi: 10.1037/0096-3445.130.4.681

Friedman, D., and Squires-Wheeler, E. (1994). Event-related potentials (ERPs) as indicators of risk for schizophrenia. Schizophr. Bull. 20, 63-74. doi: $10.1093 / \mathrm{schbul} / 20.1 .63$

Frith, C. D., and Frith, U. (2006). How we predict what other people are going to do. Brain Res. 1079, 36-46. doi: 10.1016/j.brainres.2005.12.126

Green, M. F., and Lee, J. (2012). Neural bases of emotional experience versus perception in schizophrenia. Biol. Psychiatry 71, 96-97. doi: 10.1016/j.biopsych.2011.11.005

Hajcak, G., and Foti, D. (2008). Errors are aversive: defensive motivation and the error-related negativity. Psychol. Sci. 19, 103-108. doi: 10.1111/j.14679280.2008.02053.x

Hajcak, G., and Nieuwenhuis, S. (2006). Reappraisal modulates the electrocortical response to unpleasant pictures. Cogn. Affect. Behav. Neurosci. 6, 291-297. doi: 10.3758/CABN.6.4.291

Heller, W., and Nitschke, J. B. (1998). The puzzle of regional brain activity in depres- sion and anxiety: the importance of subtypes and comorbidity. Cogn. Emot. 12, 421-447. doi: 10.1080/026999398379664

Herbener, E. S., Rosen, C., Khine, T., and Sweeney, J. A. (2007). Failure of positive but not negative emotional valence to enhance memory in schizophrenia. J. Abnorm. Psychol. 116, 43-55. doi: 10.1037/0021-843X.116.1.43

Herbener, E. S., Song, W., Khine, T. T., and Sweeney, J. A. (2008). What aspects of emotional functioning are impaired in schizophrenia? Schizophr. Res. 98, 239-246.

Hock, C., Villringer, K., Müller-Spahn, F., Wenzel, R., Heekeren, H., SchuhHofer, S., et al. (1997). Decrease in parietal cerebral hemoglobin oxygenation during performance of a verbal fluency task in patients with Alzheimer's disease monitored by means of near-infrared spectroscopy (NIRS): correlation with simultaneous rCBF-PET measurements. Brain Res. 755, 293-303. doi: 10.1016/S0006-8993(97)00122-4

Hoekert, M., Kahn, R. S., Pijnenborg, M., and Aleman, A. (2007). Impaired recognition and expression of emotional prosody in schizophrenia: review and meta-analysis. Schizophr. Res. 96, 135-145. doi: 10.1016/j.schres.2007.07.023

Horan, W. P., Blanchard, J. J., Clark, L. A., and Green, M. F. (2008). Affective traits in schizophrenia and schizotypy. Schizophr. Bull. 34, 856-874. doi: 10.1093/schbul/sbn083

Horan, W. P., Green, M. F., DeGroot, M., Fiske, A., Hellemann, G., Kee, K., et al. (2011). Social cognition in schizophrenia, part 2: 12-month stability and prediction of functional outcome in first-episode patients. Schizophr. Bull. 38, 865-872. doi: 10.1093/schbul/sbr001

Horan, W. P., Kring, A. M., and Blanchard, J. J. (2006a). Anhedonia in schizophrenia: a review of assessment strategies. Schizophr. Bull. 32, 259-273. doi: $10.1093 /$ schbul/sbj009

Horan, W. P., Green, M. F., Kring, A. M., and Nuechterlein, K. H. (2006b). Does anhedonia in schizophrenia reflect faulty memory for subjectively experienced emotions? J. Abnorm. Psychol. 115, 496-508. doi: 10.1037/0021-843X.115.3.496
Horan, W. P., Wynn, J. K., Kring, A. M., Simons, R. F., and Green, M. F. (2010). Electrophysiological correlates of emotional responding in schizophrenia. J. Abnorm. Psychol. 119, 18-30. doi: 10.1037/a0017510

Johnston, P. J., Stojanov, W., Devir, S., and Scall, U. (2005). Functional MRI of facial emotion recognition deficits in schizophrenia and their electrophysiological correlates. Eur. J. Neurosci. 22, 1221-1232. doi: 10.1111/j.1460-9568.2005.04294.x

Kameyama, M., Fukuda, M., Yamagishi, Y., Sato, T., Uehara, T., Ito, M., et al. (2006). Frontal lobe function in bipolar disorder: a multichannel near-infrared spectroscopy study. Neuroimage 29, 172-184. doi: 10.1016/j.neuroimage.2005.07.025

Kee, K., Kern, R., and Green, M. (1998). Perception of emotion and neurocognitive functioning in schizophrenia - what's the link. Psychiatry Res. 8, 57-65. doi: 10.1016/S0165-1781(98)00083-3

Kohler, C., Bilker, W., Hagendoorn, M., Gur, R., and Gur, R. (2000). Emotion recognition deficit in schizophrenia: association with symptomatology and cognition. Biol. Psychiatry 48, 127-136. doi: 10.1016/S0006-3223(00)00847-7

Kohler, C., Turner, T., Bilker, W., Brensinger, C., Siegel, S., Kanes, S., et al. (2003). Facial emotion recognition in schizophrenia: intensity effects and error pattern. Am. J. Psychiatry 160, 1768-1774. doi: 10.1176/appi.ajp.160.10.1768

Kohler, C. G., Walker, J. B., Martin, E. A., Healey, K. M., and Moberg, P. J. (2010). Facial emotion perception in schizophrenia: a meta-analytic review. Schizophr. Bull. 36, 1009-1019. doi: 10.1093/schbul/sbn192

Kono, T., Matsuo, K., Tsunashima, K., Kasai, K., Takizawa, R., Rogers, M. A., et al. (2007). Multiple-time replicability of near-infrared spectroscopy recording during prefrontal activation task in healthy men. Neurosci. Res. 57, 504-512. doi: 10.1016/j.neures.2006.12.007

Koseki, S., Noda, T., Yokoyama, S., Kunisato, Y., Ito, D., Suyama, H., et al. (2013). The relationship between positive and negative automatic thought and activity in the prefrontal and temporal cortices: a multi-channel nearinfrared spectroscopy (NIRS) study. J. Affect. Disord. 151, 352-359. doi: 10.1016/j.jad.2013.05.067

Kring, A. M., Barrett, L. F., and Gard, D. E. (2003). On the broad applicability of the affective circumplex: representations of affective knowledge among schizophrenia patients. Psychol. Sci. 14, 207-214. doi: 10.1111/1467-9280. 02433

Kring, A. M., and Elis, O. (2013). Emotion deficits in people with schizophrenia. Annu. Rev. Clin. Psychol. 9, 409-433. doi: 10.1146/annurev-clinpsy-050212185538

Kring, A. M., and Moran, E. K. (2008). Emotional response deficits in schizophrenia: insights from affective science. Schizophr. Bull. 34, 819-834. doi: 10.1093/schbul/sbn071

Kubota, Y., Toichi, M., Shimizu, M., Mason, R. A., Coconcea, C. M., Findling, R. L., et al. (2005). Prefrontal activation during verbal fluency tests in schizophrenia: a near infrared spectroscopy (NIRS) study. Schizophr. Res. 77, 65-73. doi: 10.1016/j.schres.2005.01.007

Kuwabara, H., Kasai, K., Takizawa, R., Kawakubo, Y., Yamasue, H., Rogers, M. A., et al. (2006). Decreased prefrontal activation during letter fluency task in adults with pervasive developmental disorders: a near-infrared spectroscopy study. Behav. Brain Res. 172, 272-277. doi: 10.1016/j.bbr.2006.05.020

Logothetis, N. K. (2008). What we can do and what we cannot do with fMRI. Nature 453, 869-878. doi: 10.1038/nature06976

Matsuo, K., Kato, T., Taneichi, K., Matsumoto, A., Ohtani, T., Hamamoto, T., et al. (2003). Activation of the prefrontal cortex to trauma-related stimuli measured by near-infrared spectroscopy in posttraumatic stress disorder due to terrorism. Psychophysiology 40, 492-500. doi: 10.1111/1469-8986.00051

Matsuo, K., Watanabe, A., Onodera, Y., Kato, N., and Kato, T. (2004). Prefrontal hemodynamic response to verbal-fluency task and hyperventilation in bipolar disorder measured by multi-channel near-infrared spectroscopy. J. Affect. Disord. 82, 85-92. doi: 10.1016/j.jad.2003.10.004

Mimura, M. (2014). Neuropsychiatric disorders and functional localization. Jpn. J. Neurosurg. 23, 311-317. doi: 10.7887/jens.23.311

Mocaiber, I., Sanchez, T. A., Pereira, M. G., Erthal, F. S., Joffily, M., Araujo, D. B., et al. (2011). Antecedent descriptions change brain reactivity to emotional stimuli: a functional magnetic resonance imaging study of an extrinsic and incidental reappraisal strategy. Neuroscience 193, 241-248. doi: 10.1016/j.neuroscience.2011.07.003 
Moser, J. S., Hajcak, G., Bukay, E., and Simons, R. F. (2006). Intentional modulation of emotional responding to unpleasent pictures: an ERP study. Psychophysiology 43, 292-296. doi: 10.1111/j.1469-8986.2006.00402.x

Myin-Germeys, I., Delespaul, P. A., and deVries, M. W. (2000). Schizophrenia patients are more emotionally active than is assumed based on their behavior. Schizophr. Bull. 26, 847-854. doi: 10.1093/oxfordjournals.schbul.a033499

Neuhaus, A. H., Trempler, N. R., Hahn, E., Luborzewski, A., Karl, C., Hahn, C., et al. (2010). Evidence of specificity of a visual P3 amplitude modulation deficit in schizophrenia. Schizophr. Res. 124, 119-126. doi: 10.1016/j.schres.2010. 08.014

Ochsner, K. N., Bunge, S. A., Gross, J. J., and Gabrieli, J. D. (2002). Rethinking feelings: an FMRI study of the cognitive regulation of emotion. J. Cogn. Neurosci. 14, 1215-1229. doi: 10.1162/089892902760807212

Ochsner, K. N., and Gross, J. J. (2005). The cognitive control of emotion. Trends Cogn. Sci. 9, 242-249. doi: 10.1016/j.tics.2005.03.010

Ochsner, K. N., Ray, R. D., Cooper, J. C., Robertson, E. R., Chopra, S., Gabrieli, J. D., et al. (2004). For better or for worse: neural systems supporting the cognitive down- and up-regulation of negative emotion. Neuroimage 23, 483-499. doi: 10.1016/j.neuroimage.2004.06.030

Ochsner, K. N., Ray, R. R., Hughes, B., McRae, K., Cooper, J. C., Weber, J., et al. (2009). Bottom-up and top-down processes in emotion generation: common and distinct neural mechanisms. Psycholol. Sci. 20, 1322-1331. doi: 10.1111/j.1467-9280.2009.02459.x

Ochsner, K. N., Silver, J. A., and Buhle, J. T. (2012). Functional imaging studies of emotion regulation: a synthetic review and evolving model of the cognitive control of emotion. Ann. N. Y. Acad. Sci. 1251, E1-E24. doi: 10.1111/j.17496632.2012.06751.x

Okada, F., Tokumitsu, Y., Hoshi, Y., and Tamura, M. (1994). Impaired interhemispheric integration in brain oxygenation and hemodynamics in schizophrenia. Eur. Arch. Psychiatry Clin. Neurosci. 244, 17-25. doi: 10.1007/BF02279807

Olofsson, J. K., Nordin, S., Sequeira, H., and Polich, J. (2008). Affective picture processing: an integrative review of ERP findings. Biol. Psychol. 77, 247-265. doi: 10.1016/j.biopsycho.2007.11.006

Pinheiro, A. P., Del Re, E., Mezin, J., Nestor, P. G., Rauber, A., McCarley, R. W., et al. (2013). Sensory-based and higher-order operations contribute to abnormal emotional prosody processing in schizophrenia: an electrophysiological investigation. Psychol. Med. 43, 603-618. doi: 10.1017/S003329171200133X

Regan, E. P., Rastogi, A., and Christensen, B. K. (2015). Effortful versus automatic emotional processing in schizophrenia: insights from a face-vignette task. Cogn. Emot. 29, 767-783. doi: 10.1080/02699931.2014.935297

Salem, J., Kring, A., and Kerr, S. (1996). More evidence for generalized poor performance in facial emotion perception in schizophrenia. J. Abnorm. Psychol. 105, 480-483. doi: 10.1037/0021-843X.105.3.480

Schneider, F., Gur, R., Gur, R., and Shtasel, L. (1995). Emotional processing in schizophrenia - neurobehavioural probes in relation to psychopathology. Schizophr. Res. 17, 67-75. doi: 10.1016/0920-9964(95)00 031-G

Shinba, T., Nagano, M., Kariya, N., Ogawa, K., Shinozaki, T., Shimosato, S., et al. (2004). Near-infrared spectroscopy analysis of frontal lobe dysfunction in schizophrenia. Biol. Psychiatry 55, 154-164. doi: 10.1016/S0006-3223(03) 00547-X

Shoji, Y., Morita, K., Mori, K., Yamamoto, H., Fujiki, R., Ishii, Y., et al. (2013). Characteristics of single event-related cerebral hemodynamics during verbal task in emotionally charged state measured by multi-channel near-infrared spectroscopy (NIRS) in patients with schizophrenia: comparison with healthy subjects. Seishin Shinkeigaku Zasshi 115, 853-862.

Strauss, G. P., and Gold, J. M. (2012). A new perspective on anhedonia in schizophrenia. Am. J. Psychiatry 169, 364-373. doi: 10.1176/appi.ajp.2011.11030447

Strauss, G. P., Kappenman, E. S., Culbreth, A. J., Catalano, L. T., Lee, B. G., and Gold, J. M. (2013). Emotion regulation abnormalities in schizophrenia: cognitive change strategies fail to decrease the neural response to unpleasant stimuli. Schizophr. Bull. 39, 872-883. doi: 10.1093/schbul/sbs186

Strauss, G. P., Llerena, K., and Gold, J. M. (2011). Attentional disengagement from emotional stimuli in schizophrenia. Schizophr. Res. 131, 219-223. doi: 10.1016/j.schres.2011.06.001

Suto, T., Fukuda, M., Ito, M., Uehara, T., and Mikuni, M. (2004). Multichannel near-infrared spectroscopy in depression and schizophrenia: cognitive activation study. Biol. Psychiatry 55, 501-511. doi: 10.1016/j.biopsych.2003.09.008

Taylor, S. T., Kang, J., Brege, I. S., Tso, I. F., Hosanagar, A., and Johnson, T. D. (2012). Meta-analysis of functional neuroimaging studies of emotion perception and experience in schizophrenia. Biol. Psychiatry 71, 136-145. doi: 10.1016/j.biopsych.2011.09.007

Walker, E., Maguire, M., and Bettes, B. (1984). Recognition and identification of facial stimuli by schizophrenics and patients with affective disorders. Br. J. Clin. Psychol. 23, 37-44. doi: 10.1111/j.2044-8260.1984.tb00624.x

Wynn, J. K., Jahshan, C., Altshuler, L. L., Glahn, D. C., and Green, M. F. (2013). Event-related potential examination of facial affect processing in bipolar disorder and schizophrenia. Psychol. Med. 43, 109-117. doi: $10.1017 /$ S0033291712001006

Conflict of Interest Statement: The authors declare that the research was conducted in the absence of any commercial or financial relationships that could be construed as a potential conflict of interest.

Copyright (C) 2015 Balconi, Tirelli and Frezza. This is an open-access article distributed under the terms of the Creative Commons Attribution License (CC BY). The use, distribution or reproduction in other forums is permitted, provided the original author(s) or licensor are credited and that the original publication in this journal is cited, in accordance with accepted academic practice. No use, distribution or reproduction is permitted which does not comply with these terms. 